\title{
3.3 La revisión por pares iguales: un instrumento de innovación educativa
}

Rita Tejada, Ph.D.

\section{Resumen}

Este artículo trata sobre la revisión por pares iguales, su definición y su utilización como un método de enseñanza-aprendizaje en la práctica de la escritura en todas las disciplinas enseñadas a nivel superior. Se señalan las ventajas y desventajas de este proceso y se ofrecen modelos para implementarla y evaluarla en el salón de clase. Palabras claves: revisión por pares iguales (peer review); trabajo en grupo, aprendizaje por observación.

Cuando se habla de innovación educativa enseguida pensamos en la innovación tecnológica que hoy en día permea todas las instancias de nuestra vida. El uso de tecnología en nuestros salones de clase implica una interacción más inmediata entre el docente y una máquina, de la índole que ésta sea. Esto quizá nos hace mirar de soslayo y hasta subestimar la innovación que tiene lugar utilizando el elemento primario de la actividad educativa: los estudiantes. Uno de los instrumentos usados para promover la interacción entre estudiantes y potenciar sus habilidades en clase es la revisión por pares iguales o peer review.

Considerado como un método para mejorar la calidad de los trabajos escritos, la revisión por pares iguales se ha empleado en las revistas científicas especializadas (en áreas como psicología, ciencias informáticas, física, química, astronomía, por sólo nombrar algunas), para seleccionar los trabajos que se les suministran para posible publicación. En este proceso, el ensayo es leído por varios especialistas de la profesión (su número oscila entre dos y seis), quienes hacen comentarios y recomiendan o rechazan su publicación. Aunque la revisión por pares en las publicaciones puede considerarse un proceso evaluativo, la aplicación de esta modalidad en las clases que requieren trabajos escritos no se contempla como el resultado final de una evaluación, sino como una parte del proceso de enseñanza-aprendizaje donde los estudiantes se valen de sus compañeros de clase para mejorar la calidad de su escritura y / o cualquier trabajo que requiera creatividad por parte del discente.

Crespo et al. (2004) indican el uso de la revisión por pares iguales en instituciones educativas desde el año 1995.

Entre las desventajas de este método, se indican las siguientes:

a) Las revisiones toman el tiempo de la clase.

b) En su papel como revisores, los estudiantes no hacen comentarios útiles.

c) Los estudiantes necesitan más tiempo que un período de clase para leer los trabajos con cuidado.

d) Los estudiantes no hacen correcciones de estilo ni de gramática;

e) Las revisiones no son imparciales.

Entre las ventajas del uso de la revisión por pares iguales se indican que ésta:

1 Dra. Literatura y Profesora Asistente del Depto. de Lenguas Modernas y Literatura Luther College, Iowa, Estados Unidos, tejadari@luther.edu, (563) $387-1127$ 
a) Beneficia al estudiante por los comentarios y/o sugerencias que le proveen sus compañeros de clase.

b) Potencia las habilidades críticas de los estudiantes.

c) Garantiza un feedback inmediato.

d) Incentiva al estudiante a "aprender por observación" (learning by observation) (Crespo et al., p. 2), ya que tiene que leer los trabajos de sus otros compañeros.

e) Aumenta la motivación, surgida del espíritu de competencia entre los estudiantes.

f) Permite que el estudiante someta el borrador de su trabajo para revisión $\mathrm{y}$, entonces, tenga la oportunidad de entregar a su profesor un trabajo de más calidad

g) Incentiva la interacción entre los estudiantes, no importa el tamaño de la clase, al conformar a estudiantes y revisores en grupos pequeños.

h) Utiliza una estructura diferente a la tradicional de asignar trabajos que el profesor sólo tiene tiempo de corregir al final de un ciclo o semestre.

i) Divide la responsabilidad, ya que tanto el estudiante como sus revisores serán responsables; el primero, de incorporar aquellas sugerencias que mejorarían su trabajo y los últimos, de hacer una revisión seria.

j) Elimina la jerarquización, pues tanto estudiantes como revisores estarán al mismo nivel en el salón de clase.

k) Facilita al instructor el trabajo de corrección final.

1) Mejora la habilidad escrita de los discentes.

Aunque colocada en el último lugar de la lista, la escritura tiene que promo- verse en todas las disciplinas de estudio para formar futuros profesionales cuyos trabajos les exigirán esta habilidad. El objetivo de incentivar la escritura en las clases puede lograrse mediante el uso de diversos formatos: una revisión bibliográfica, una narración personal, una bibliografía anotada, una descripción, un memorando, una explicación de un proceso, un artículo para una revista o periódico, un reporte sobre una experiencia personal o tarea asignada, un resumen de un libro o ensayo, una carta o un esquema para desarrollar un proyecto (Fassler Walvoord, p.10).

Una manera de implementar la revisión por pares en las clases puede empezar con una estructura como la siguiente:

1) Los estudiantes redactan un escrito breve, digitado en computadora y a doble espacio (1 ó 2 páginas).

2) A medida que van llegando a la clase, los estudiantes ponen su trabajo en el escritorio del profesor y toman el escrito de otro estudiante.

3) Cada estudiante escribe un comentario o señala algún aspecto que necesita modificarse en el trabajo de su compañero/a y lo firma con su nombre.

4) Los estudiantes forman grupos (de 2, 3 hasta un máximo de 4 estudiantes) y van intercambiando papeles hasta que todos los miembros del grupo lean y comenten todos los trabajos;

5) Los trabajos son entregados al profesor, quien los devuelve a sus respectivos dueños.

Los estudiantes son instruidos entonces para que lean los comentarios, hagan las modificaciones pertinentes y entreguen las dos copias del trabajo a su profesor. El 
docente podrá comparar los dos trabajos y determinar cuáles cambios se han producido. La primera copia del trabajo también ayudará al docente a evaluar la habilidad crítica de sus estudiantes con el propósito de, eventualmente, poder agrupar a sus discentes según dichas habilidades.

Crespo et al. han establecido un sistema de perfiles para maximizar el proceso de aprendizaje mediante la revisión por pares iguales. Ellos sugieren la asignación de trabajos cortos a ser revisados por pares iguales, lo que permitirá la creación de un archivo de perfiles sobre los estudiantes en su papel como revisores. Estos investigadores indican que un estudiante con dificultades en sus trabajos se beneficia grandemente cuando la contraparte del proceso de revisión por pares iguales es un buen estudiante. El estudiante habilidoso se sentirá estimulado para hacer sugerencias y comentarios al trabajo de su compañero, mientras el estudiante con dificultades se beneficiará del proceso mediante el "aprendizaje por observación" al leer el trabajo de un buen estudiante.

Si bien la revisión por pares iguales estimula el intercambio, este método no es una camisa de fuerza, ya que "confiere a los estudiantes el poder de decidir sobre la validez de las sugerencias de sus revisores" (Nelson, p. 3). Es decir, es el estudiante quien al final incorpora o no lo que en el proceso de revisión le ha sido señalado.

El/la profesora puede proveer al estudiante con un esquema de preguntas, cuyos criterios debe tomar en cuenta para asumir su papel de revisor con responsabilidad. Un esquema puede ser el siguiente, referido a la revisión de un breve artículo de opinión:
Revisor/a

Título del trabajo:

1. En una oración, describe el problema que trata este artículo:

2. Resume cuál es la opinión (punto de vista) del/ de la autora:

3. Con un marcador, colorea las partes del texto que te parecen confusas.

4. Nota: Si no puedes establecer claramente los aspectos pedidos en 1 y 2 , escribe a continuación algunos comentarios que ayudarían a clarificarlos:

5. ¿Este artículo sustenta su opinión con bibliografía, estadísticas o ejemplos? ¿Crees que tu compañero/a necesita investigar más? ¿Por qué?

6. ¿Detectas errores de gramática $(\mathrm{g})$, ortografía

(o) o puntuación (p) que deban corregirse? Señálalos en el trabajo.

7. Escribe una sugerencia que ayude a mejorar este artículo:

La revisión por pares iguales también puede adaptarse para trabajos en grupo. Por ejemplo, el diseño de una página web en una clase de informática se puede proponer como un proyecto entre cuatro estudiantes. A cada estudiante se le asignará una parte. Los integrantes del equipo se encargarán de revisar cada asignación antes de entregar el trabajo final. También, cada proyecto puede someterse a revisión por otros equipos de estudiantes. La ventaja de esta actividad generará una competencia indirecta que llevará a los estudiantes a sentirse más motivados y a producir mejores proyectos. Para hacer más eficiente el manejo del tiempo dentro y fuera de la clase, con estos proyectos

\section{Cuaderno de Pedagogía Universitaria}


se puede recurrir al uso de los foros que proveen los CMS (Course Management Systems). La incorporación y combinación de la tecnología ayudaría aún más a incrementar la escritura, propósito principal de la revisión por pares iguales.

A la revisión por pares iguales también se le puede añadir un elemento de evaluación. Por ejemplo, en el esquema de la revisión por pares iguales del artículo de opinión, los estudiantes que reciban los comentarios pueden "evaluar" a su revisor al añadirse un ítem como el siguiente:

Evaluación del/de la revisor/a:

a. Considero que el trabajo de revisión hecho por mi compañero/a fue:

5) excelente

4) muy buena

3) buena

2) regular

1) mala

b. Explica tu evaluación:

De lo anterior podemos inferir que la revisión por pares iguales es un método bastante flexible y adecuado para la enseñanza-aprendizaje a nivel superior. Su flexibilidad lo hace fácilmente adaptable a los diferentes esquemas de las clases universitarias que busquen no sólo mejorar la escritura sino también promover mayor interacción entre los estudiantes, principio $\mathrm{y}$ fin del quehacer educativo.

\section{Bibliografía}

BARRY, Nancy, KILDEGAARD, Lise (2005). Using Writing Exercises and Learning Strategies in the New 60/90 Minute Schedule. Faculty Development Workshop, Friday August 26, 2005, Luther College, Iowa

BEAN, John C. (1996). Engaging Ideas. The Professor's Guide to Integrating Writing, Critical Thinking, and Active Learning in the Classroom. San Francisco, Jossey-Bass Publishers.

CRESPO, Raquel M., PARDO, Abelardo, DELGADO KLOOS, Carlos (2004). An Adaptive Strategy for Peer Review. 34th ASEE/IEEE Frontiers in Education Conference, October 20-23, 2004, Savannah, GA http://fie.engrng. pitt.edu/fie2004/papers /1356.pdf [Consulta:sept. 2005]

NELSON, Stephanie (2000). Teaching Collaborative Writing and Peer Review Techniques to Engineering and Technology to Undergraduates, en 30th ASEE/IEEE Frontiers in Education Conference, October 18-21, 2000, Kansas City, MO <http://fie. engrng.pitt.edu / fie2000/ papers / 1422. pdf $>$ [Consulta:sept. 2005]

WALVOORD, Barbara E. Fassler (1982). Helping Students Write Well. A Guide for Teachers in All Disciplines. New York, The Modern Language Association of America. 